\title{
Effects of different wavelength from Light Emitting Diodes (LEDs) on growth and development in zebrafish (Danio rerio) embryos and larvae
}

\author{
Shafira Septriani ${ }^{1, *}$, Anley Teferra Kiddane ${ }^{2}$, Gun Do Kim², and Christopher L. Brown ${ }^{1}$ \\ ${ }^{1}$ Division of Fisheries Science, The Graduate School of World Fisheries University, Pukyong National \\ University, Busan, Republic of Korea \\ ${ }^{2}$ Department of Microbiology, College of Natural Science, Pukyong National University, Busan, \\ Republic of Korea
}

\begin{abstract}
A light environment in aquatic habitat is one of the essential physical parameters affecting the growth and physiology of fish. To investigate the effects of light on the growth of zebrafish embryos and larvae (Danio rerio), fishes were reared under the illumination of Light Emitting Diodes (LEDs) of different wavelengths, including green, red, blue, and white. Morphometric, neuromast staining with 2-[4(Dimethylamino)styryl]-1-ethylpyridinium iodide (DASPEI) and Polymerase Chain Reaction (PCR) analyses were used to examine the initial development of sensory capacity in this study. On days 5,6, and 7, the green light produced the most favorable result. Blue light consistently impacted early parameters such as eye development, egg yolk absorption, and total length. These impacts were synchronous with the initiation of feeding. Exposure to specific wavelengths of light can induce the appearance of gene expression, opn 1 lw1 detected opsin and (mtnrlba and aanat2) were active under all four experimental lighting conditions. This retinal gene appears to be sensitive to ambient wavelength, but apparently, genes in the melatonin pathway are not.
\end{abstract}

\section{Introduction}

Zebrafish (Danio rerio) is a small freshwater fish popular as a tropical ornamental aquarium pet $[40,24]$. Wild zebrafish are abundant schooling fish occupying a wide range of freshwater habitats in both slow-flowing rivers found in relatively small groups or schools of up to 300 individuals [41]. Zebrafish has been commonly used worldwide as an object of research, especially over the past few decades $[39,18]$. Zebrafish are accessible to culture in captivity and breeding $[25,24]$.

Growth is described as an increase in the size of the body over time. Some benchmarks can be used as a basis for measuring growth, such as measuring the length and weight of objects per time, the percentage of weight gain, or body dimensions per time [26]. Growth is

\footnotetext{
* Corresponding author: brownchristopher38@gmail.com
} 
marked by the development of progressively well-functioning body cells. Various regulatory and other factors control the growth of fish. The main factors impacting growth in aquaculture are feed consumption, stocking density, biotic factors (sex, age, genetic), and abiotic factors (water quality: temperature, $\mathrm{pH}$, salinity, dissolved oxygen, organic compounds, and light [26]. Another factor to affect growth is the family of regulatory compounds, including growth hormones $(\mathrm{GH})$, which control metabolic activities [9]. Growth-associated physiological processes include stress responses, feeding, and reproduction in fish [7]. Most growth promotion by GH is mediated by hepatic or locally produced Insulin-Like Growth Factor 1 (IGF-1) [7].

Larval fish have different physiology and ecology and are sensitive to environmental and ecological pressures that differ from those endured by adults. During early embryo and larval development, tolerance of variable abiotic factors is relatively narrow [2]. From embryo development to sexual maturation, the early life of fish is profoundly affected by light [3]. Light is a regulatory factor that affects the growth and survival of juvenile fish $[12,19]$.

Sunlight is the primary light source for wild zebrafish, and there are a few secondary sources such as moonlight, starlight, and luminescent organisms. Artificial light is mainly used in aquaculture operations, typically with halide, neon, or light-emitting diode (LED) light sources. Light is highly variable and can change over an enormous range, often very quickly. Light is a complex environmental factor that can vary in intensity and spectrum, daylength (or photoperiod), water visibility, and specific light sensitivities of the species being reared $[7,48]$.

Experimental evidence demonstrates that the color or wavelength of light strongly influences zebrafish embryo development under their reared [49]. Light has regulatory effects in fish on the synthesis of pituitary growth hormone, indirectly affecting the feeding and survival of fish, and light-induced stress conditions are associated with impaired immune function, resulting in deterioration of growth and maturation [31].

Another effect of light is the promotion of retina development. The retina lies at the back of the eye. It is a sensory component of the central nervous system (CNS), consisting of six neural cells and one glial cell laminated into three distinct nuclear layers [50]. The retina can adapt to various light conditions. However, prolonged exposure to intense light can cause photoreceptor cell damage [33]. Similarly, light can lead to physiological stress responses and may appear to change behavior, reduce appetite, with resulting impacts on the health and growth of fish $[38,14]$.

LED light sources to offer superior light intensity, efficiency, and small size when used as the aquarium tank's light resource and are recently famous in the aquaculture industry [38, 53]. LED provides specific wavelengths of light and intensity to be manipulated to simulate the environmental conditions to those acceptable with target organisms [12]. Red or long spectrum penetration in shallow water negatively impacts some physiological functions of fish [19]. Survival of larval fish has an extensive range of responses to the wavelength of ambient light, depending on the species and rearing condition [48].

The present study examined the impacts of variable wavelengths of light produced by LEDs on zebrafish embryos and larvae development. Recent investigations have demonstrated that different wavelengths of light can elicit various physiological responses in fish [19]. [10], reported that low wavelength light resulted in physiological stress in olive flounder (Paralichthys olivaceus) during starvation. Another study [19] revealed that rainbow trout grown under different wavelengths of light had statistically significant differences in antioxidant enzyme activities in the liver, brain, and gill. 


\section{Materials and methods}

\subsection{Animal rearing condition}

This research was conducted at the World Fisheries University at Pukyong National University, Busan, South Korea, from August 2020 to February 2021. Wild-type zebrafish were purchased from a local supplier and reared for five months, following the general guidelines of [32]. Zebrafish broodstock colony was separated by gender and maintained in a recirculating tank of dechlorinated water between $23-25^{\circ} \mathrm{C}$ in 100 liters. Fishes were fed with TetraMin flake feed and Artemia sp. nauplii (newly hatched brine shrimp) twice daily, photoperiod was maintained with a timer set at LD 14:10 (14 hours of light and 10 hours of darkness per day).

\subsection{Experimental design}

\subsubsection{Eggs collection and general experimental conditions}

Spawning tanks were set up with two pairs of zebrafish broodstock with six spawners in each spawning tank, using a ratio of 2 females to each one male, tank measuring $15 \mathrm{~cm} \mathrm{x} 10 \mathrm{~cm}$ placed in the aquarium tank. Fertilized eggs were collected after 48 hours maximum. Eggs were selected with the perfect shape used as a treatment in this research. Dead and physically damaged eggs were removed, and only developing fertilized eggs were used and placed into experimental units.

\subsubsection{LED light simulator set}

LEDs of different colors: red, green, blue, and white lamps, were used for experimental treatments. The LED was attached to encircle the bottom of the quadrangular shape box with dimensions: $28.5 \mathrm{~cm} \times 23.5 \mathrm{~cm} \times 10.5 \mathrm{~cm}$ and has a hole for the cable connected with an automatic switch timer. At the bottom of the box was placed one well plate consisting of 6 wells, each with a diameter of $34.8 \mathrm{~mm}$ and a volume of $16.8 \mathrm{~mL}$. Eggs were transferred and counted using a wide-mouth pipette to the experiment plate, then filled with culture water. The stocked density was 15 eggs per well plate, and the total number of larvae used in this experiment was 90 per each experimental light treatment. Every day the water level in each well was controlled, and culture water was added to replace water lost to evaporation. The room temperature was maintained at $27^{\circ} \mathrm{C}-28^{\circ} \mathrm{C}$, which is considered to be approximately optimal for larval zebrafish growth [43].

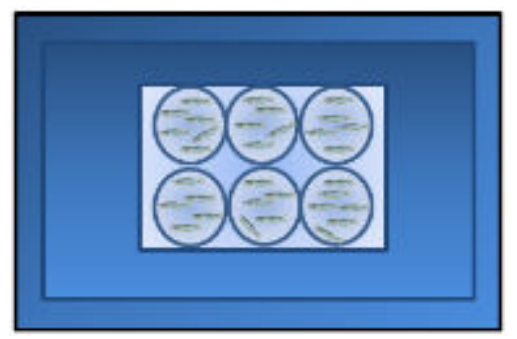

Fig. 1. Light simulator box. 


\subsubsection{Light treatment}

In this experiment, the embryos and larvae were exposed to light-emitting diodes (LEDs) respectively green, blue, red, and white with the intensity of 750 Lux, under LD 14:10 photoperiod exposure. This light intensity has been used in the studies of light regimes on larval performance, and the LD 14:10 photoperiod is considered standard for reproductively active zebrafish [1]. Light spectra were varied according to wavelengths used by [49], blue $472 \mathrm{~nm}$, green $436 \mathrm{~nm}$, red $665 \mathrm{~nm}$, and white 668nm (Fig. 2). Experimental observations were continued from day one up to 10 days after fertilization (dpf), monitored daily for developmental changes by quantifying parameters as specified in section 2.3.3.5.

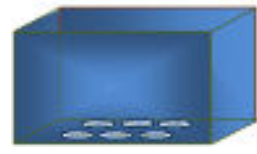

Blue

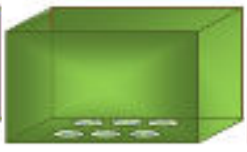

Green

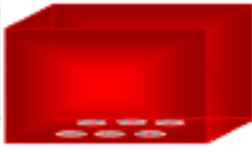

Red

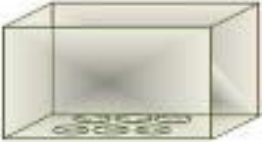

White

Fig. 2. Light treatment set.

\subsection{Sampling procedures}

\subsubsection{Survival and viability}

After 24 hours, deformed and moribund larvae were counted and removed. Viable hatch was determined as the percentage of the total hatch that was alive and normally developed from 1 dpf until ten dpf. Total mortality represented the combined percentage of dead eggs, abnormal fry, and dead and moribund larvae.

\subsubsection{Larva traits}

Larval traits for some categories were quantified, such as time to hatch larvae, pericardial area, body length, tail length, eye, and swimbladder size. The pericardial area was calculated by circling the pericardial space using conventional image software (Fiji Image J). Body total length is the length from the tip of the mouth until the last spike of the tail of larvae. The length of the tail (Fig. 3a) was measured from the first somite (anterior part) to the finished part of the posterior [15]. Tail curvature formation (normal, lordosis, and kyphosis) was noted. Measurement of eye size was carried out diagonally throughout the eye. Measuring the length of the swimbladder was conducted.

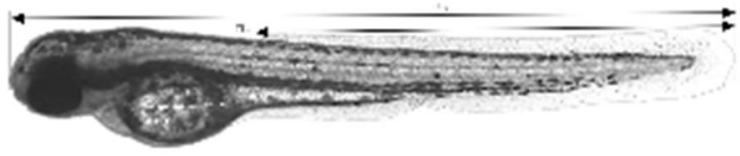

(a)

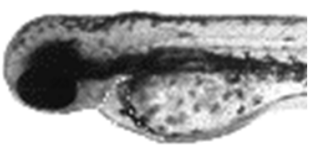

(b)

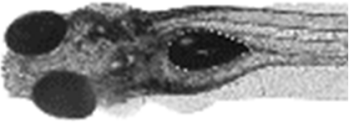

(c)

Fig. 3. Zebrafish Larva Measurement. $\mathrm{TL}_{1}$ : total length, $\mathrm{TL}_{2}$ : tail length, EYD: (a) egg yolk diameter, eye diameter, (b) pericardial area, (c) swimbladder area. 


\subsubsection{DASPEI staining}

DASPEI is a voltage-sensitive dye that causes fluorescence in cells with active mitochondria. It is frequently used to assess the status of hair cells of the lateral line neuromasts and the nasal epithelium of the fish [30]. It has also been used to indicate the maturational status of larval fish intestinal tracts [23]. DASPEI staining causes functional neuromast cells to fluoresce under the microscope. Sample larval zebrafish were immersed in DASPEI with a concentration of $6 \mathrm{ppm}$. An amount of $0.18 \mathrm{mg}$ DASPEI was diluted with $30 \mathrm{~mL}$ of distilled water to prepare a stock solution. Zebrafish larvae were immersed for 3 hours and then examined microscopically following the method [23]. The number of neuromasts seen was counted and compared between treatments and controls.

\subsubsection{Polymerase Chain Reaction (PCR) analysis}

Measurements of the effect of different wavelengths of light on transcription opn 1lw1, $m t n r 1 b a$, and aanat 2 genes were quantified in embryos and in 5 dpf larvae, using wholebody homogenates for PCR analysis, and the results are descriptive analysis. Collectively larvae were pooled to a weight of around 20-100 mg per sample. Step to PCR analysis consists of DNA extraction, PCR, electrophoresis, PCR purification, and sequencing. All PCR methods are more fully explained in the appendix.

Table 1. Primer used for PCR.

\begin{tabular}{ll}
\hline \multicolumn{1}{c}{ Gene } & \multicolumn{1}{c}{ Primer 5' to 3' } \\
\hline opn1lw1 (LWS-1) & Forward: CCC-ACA-CTG-CAT-CTC-GAC-AA \\
& Reverse: AAG-GTA-TTC-CCC-ATC-ACT-CC \\
\hline mtnrlba & Forward: CAT-TGG-TCC-CTG-ATT-GGC-TG \\
& Reverse: GTC-CCG-CCT-TTT-GAT-GTC-TC \\
\hline aanat2 & Forward: AGG-ACG-CCA-TCA-GTG-TGT-TT \\
& Reverse : CTG-GCC-CAG-GAA-AAC-AAG-TA \\
\hline
\end{tabular}

pnllw1 (lws-1) : red sensitive opsin 1

mtnrlba: melatonin receptor type $1 \mathrm{~B}-\mathrm{A}$

aanat 2 : arylalkylamine $\mathrm{N}$-acetyltransferase

\subsection{Statistical analysis}

Data analysis was carried out to determine the reactions to the differences in color of the lights with each of the parameters specified. The results were then statistically analyzed to compare the significance of apparent differences between treatments. All statistical tests were done using Graph Pad Prisma, version 9. First, the data were checked with two-way ANOVA with multiple comparisons and expressed with a mean \pm SEM data) between the time groups. Further analysis of data was carried out using a Tukey's test $(p<0.05)$. The mean values ( $p$ value) indicated by different superscripts were significantly different. One superscript suggests a relatively weak but significant difference ( $p$-value $<0.05$ ), two superscripts indicate a medium difference ( $\mathrm{p}$-value $<0.005$ ), and three superscripts indicate an enormously significant difference ( $\mathrm{p}$-value $<0.001)$.

\section{Results}

The survival rate was calculated by counting the number of larvae remaining minus eggs that died from infertility and the larvae that died during the experiment period (Fig. 4.). On the 
first day, the survival rate sharply decreased, the frequency of mortality in all treatments was very high, marked with white eggs. Survival was ascertained for the first ten dpf. The highest survival rates were observed under green and white light, but the pattern suddenly decreased on the $7^{\text {th }}$ day below the white light exposure, with a more consistent display of a way of survival in green light.

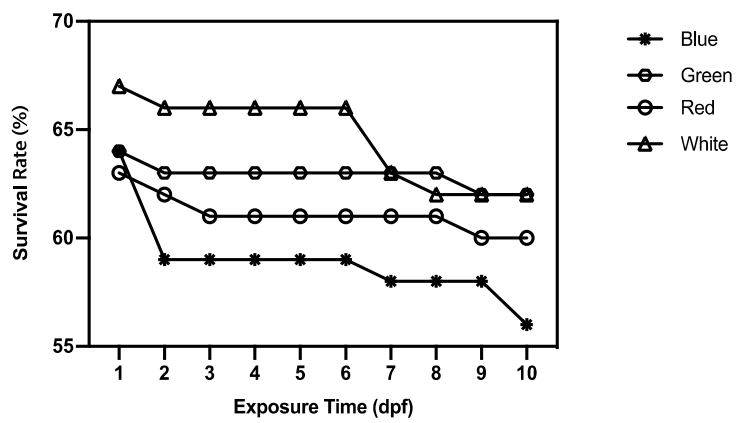

Fig. 4. Survival Rate (\%) from $1^{\text {st }}$ up to $10^{\text {th }}$ day post fertilization.

Several morphometric parameters were used to determine the wavelengths that have a positive or negative effect on the development of zebrafish larvae. Morphometric measurement is a mathematical measure, interpretation, and analysis to quantify variability in size during development. This technique describes the shape of structures by comparatively measuring the morphology of organ systems [37]. Fiji Image $\mathrm{J}$ application was used to convert images from pixel size to millimeters.

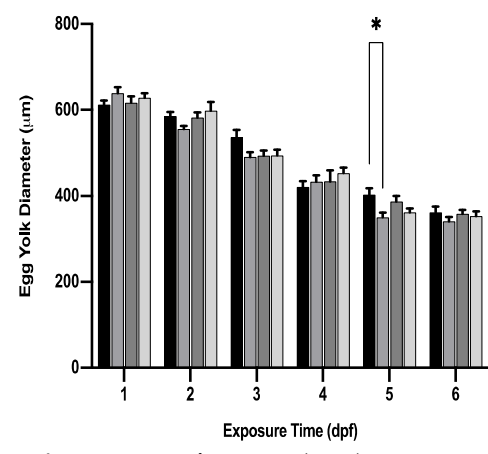

Fig. 5. Egg Diameter (mm). Data are expressed as means \pm SEM
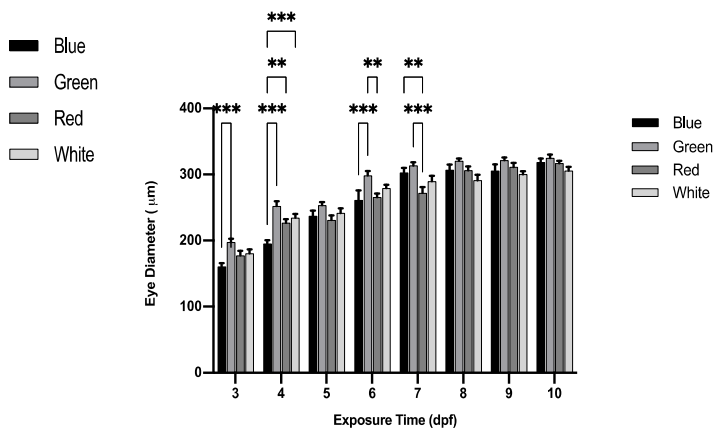

Fig. 6. Eye Diameter $(\mu \mathrm{m})$, Data are expressed as means \pm SEM

Although no significant differences were detected, the size of the zebrafish eye diameter under green light exposure had the highest mean value compared to other treatments. In contrast, the white light exposure had the lowest $(\mathrm{p}<0.05)$.

The single highly significant difference between treatments was observed on day seven pdf between blue $36.83 \pm 4.87$ and green $64.33 \pm 5.63$ swimbladder areas expressed as mean + SEM, with very significantly different values $(\mathrm{p}<0.001)$. In general, on day ten, green had the highest mean value of $76.06 \pm 2.25$ in contrast with white light exposure, which had the lowest value of $62.61 \pm 4.17$. 


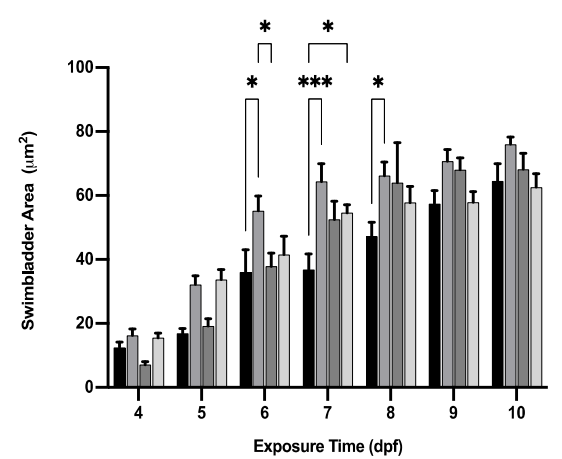

Fig. 7. Swimbladder Area $\left(\mu \mathrm{m}^{2}\right)$, Data are expressed as means \pm SEM from day 4-10.

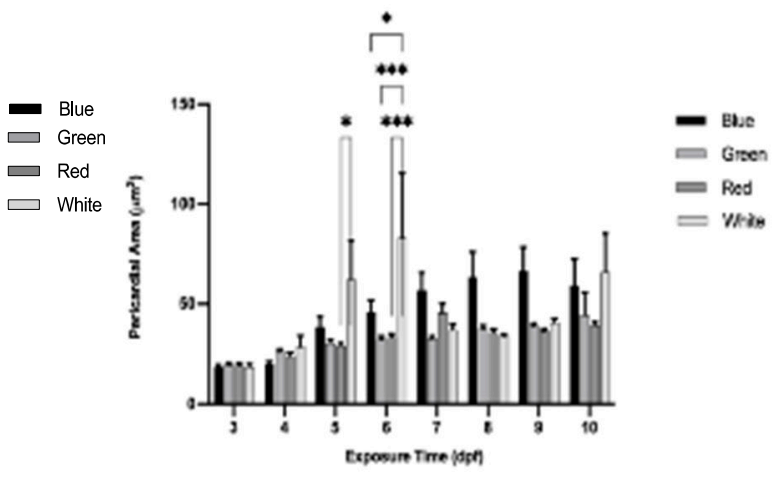

Fig. 8. Pericardial Area $\left(\mu \mathrm{m}^{2}\right)$. Data are expressed as means \pm SEM from day 3-10.

As seen in Fig. 8., a change in the size of the pericardial area on day 5 and 6 were observed under white light exposure due to several larvae with abnormal pericardial edema in randomly sampled specimens. From the seventh day onwards, there was a decrease in the size of the edema due to larval mortality. This result follows the decreased survival rate on day 7.

Tail length and total length results are shown in Fig. 9. and 10. There are similarities between the two graphs, and a significant difference was observed starting from days 4, 5, 6 to $7 \mathrm{dpf}$. However, in general, the enormous total lengths are green, white, red, and blue.

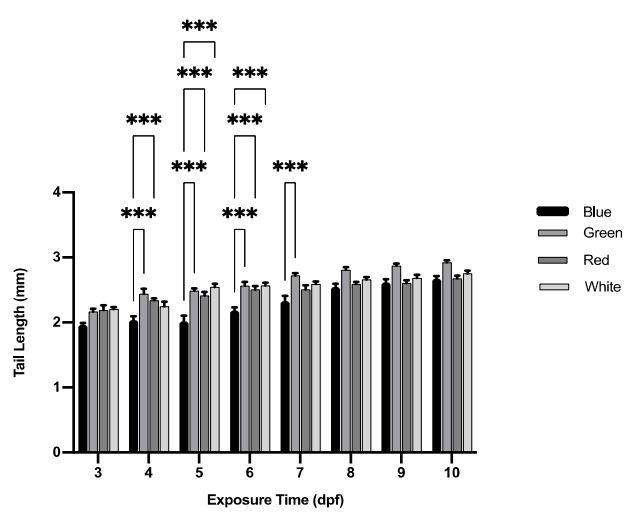

Fig. 9. Tail Length (mm). Data are expressed as means \pm SEM from day 3-10.

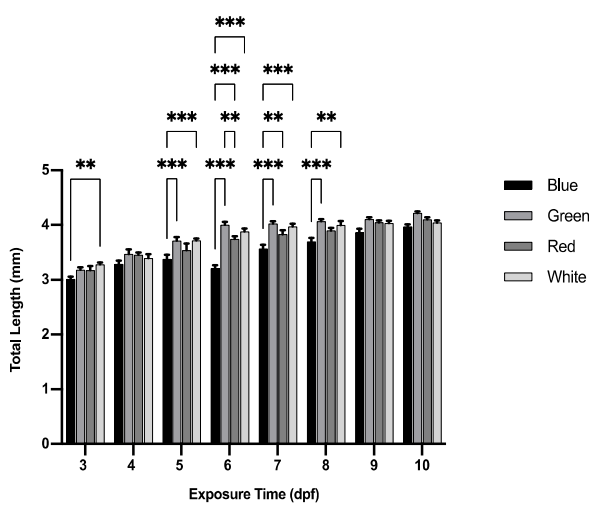

Fig. 10. Total Length (mm). Data are expressed as means \pm SEM from day 3-10.

Embryo development of zebrafish under green light exposure (Fig. 11.b) was more rapid than other treatments, as clearly reflected in the earlier development of eye pigmentation, among other parameters. Other zebrafish embryos at $2 \mathrm{dpf}$ had evidence of abnormalities shown by the black arrow. There was a decrease in the rate of hatching of zebrafish embryos exposed to blue light associated with morphological defects (yellow edema, pericardial edema, spine and tail deformity) shown in Fig. 11. 


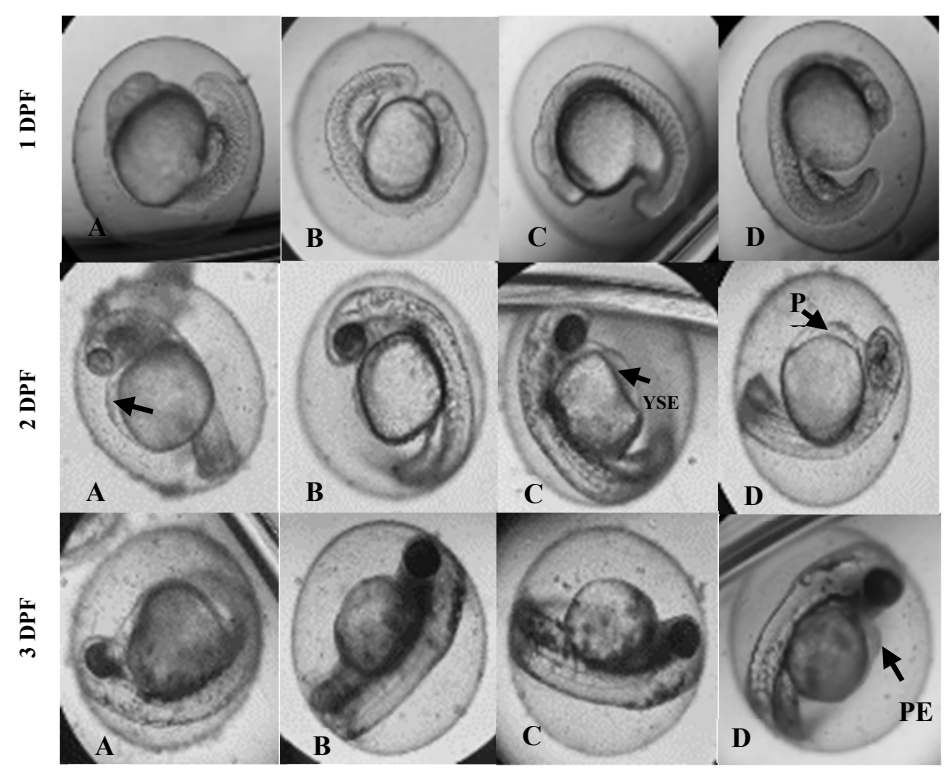

Fig. 11. Development of Zebrafish Embryos, A: blue, B: green, C: red, D: white from $1^{\text {st }}-3^{\text {rd }}$ DPF (day post fertilized. PE indicates pericardial edema, YSE indicates yolk sac edema showed with arrow in the picture.

The effect from environmental wavelength and eye development in embryonic zebrafish are presented in Table 2.

Table 2. Eyes development during embryogenesis from 24 to $72 \mathrm{hpf}$.

\begin{tabular}{|l|c|c|c|}
\hline \multirow{2}{*}{ Treatments } & \multicolumn{3}{|c|}{ Exposure Time (hpf) } \\
\cline { 2 - 4 } & $\mathbf{2 4 ~ h}$ & $\mathbf{4 8 ~ h}$ & $\mathbf{7 2 ~ h}$ \\
\hline Blue & - & + & ++ \\
\hline Green & - & ++ & +++ \\
\hline Red & - & ++ & +++ \\
\hline White & - & + & +++ \\
\hline
\end{tabular}

Where:

(-) unpigmented and undeveloped; $(+)$ slightly pigmented; $(++)$ medium pigmented; $(+++)$ fully pigmented. hpf : hour post fertilized

Differences in the embryo's development in response to the different wavelengths can be seen based on the pigmentation in the fish's eyes. At $24 \mathrm{hpf}$, eye formation was seen but not yet fully differentiated and still unpigmented, $48 \mathrm{hpf}$ there was a difference. It was apparent that blue and white light inhibited the development of eye pigmentation. At $72 \mathrm{hpf}$ only embryos in blue light exposure experienced inhibition of eye pigmentation and opposite responses to at green, red and white wavelengths. However, it should be noted that from 36 samples taken at each treatment, the results obtained at $72 \mathrm{hpf}$ of eye pigmentation in the zebrafish larva embryo were fully pigmented with a value of $99 \%$ under green exposure $91 \%$ under red exposure, and $89 \%$ under white exposure. 

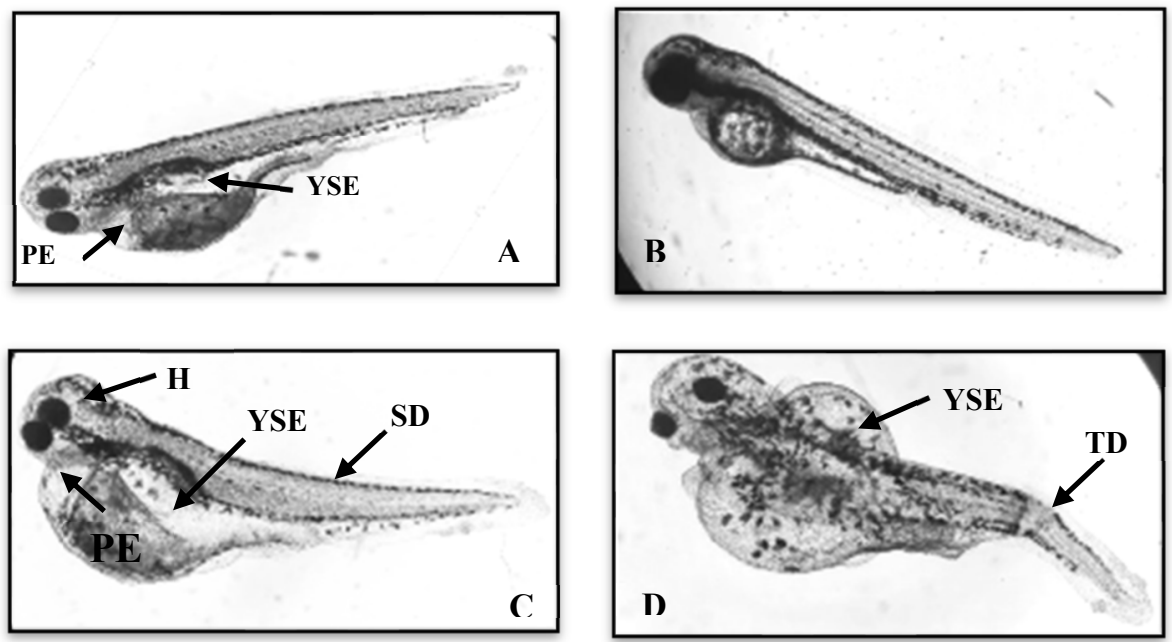

Fig. 12. Zebrafish Larva 4 dpf Abnormalities, A: blue, B: green, C: red, D: white, abnormalities $\mathrm{PE}$ indicates pericardial edema, YSE indicates yolk sac edema, HC indicates hydrocephalus, SD indicates spinal deformity and TD indicates tail deformity marked

The lateral line is a hydrodynamic sensory system found in all fishes that enables feeding, shoaling, and communication behavior [11]. It generally matures during larval development. As revealed with DASPEI staining, the lateral line illustrates the maturation of neuromasts present in each larva in response to different light exposure conditions. The immersion of 10day-old larvae in DASPEI within 3 hours resulted in the staining of neuromasts (hairlines). Immersion in the stain material for more than 3 hours results in stain saturation, and the status of the neuromast cannot be observed in this condition. Neuromasts are scattered from the head to the tip of the tail on the skin surface in Fig. 13. Neuromast consists of small patches. This Fig.13 indicated glowing green spots forming a straight line. It can also be observed in Fig. 13. that the digestive system of all treatments has active mitochondria (in other words, is metabolically functioning) with a positive response to fluorescent staining with DASPEI. According to Table 3., there was no significant difference in the results of neuromast staining using 2- [4- (Dimethylamino) styryl] -1-ethylpyridinium iodide (DASPEI). However, despite the lack of statistical significance, the highest mean number of fluorescents neuromast cells in response to green light exposure is followed by red, white and blue.

Table 3. Total Neuromast in Each Treatment. Data are expressed as means \pm SEM $(p<0.05)$.

\begin{tabular}{|c|c|c|c|c|}
\hline \multirow{2}{*}{ means \pm SEM } & \multicolumn{4}{|c|}{ Treatments } \\
\cline { 2 - 5 } & Blue & Green & Red & White \\
\cline { 2 - 5 } & $36.67 \pm 3.84^{\mathrm{a}}$ & $39.33 \pm 4.06^{\mathrm{a}}$ & $38.67 \pm 3.18^{\mathrm{a}}$ & $38.33 \pm 5.21^{\mathrm{a}}$ \\
\hline
\end{tabular}



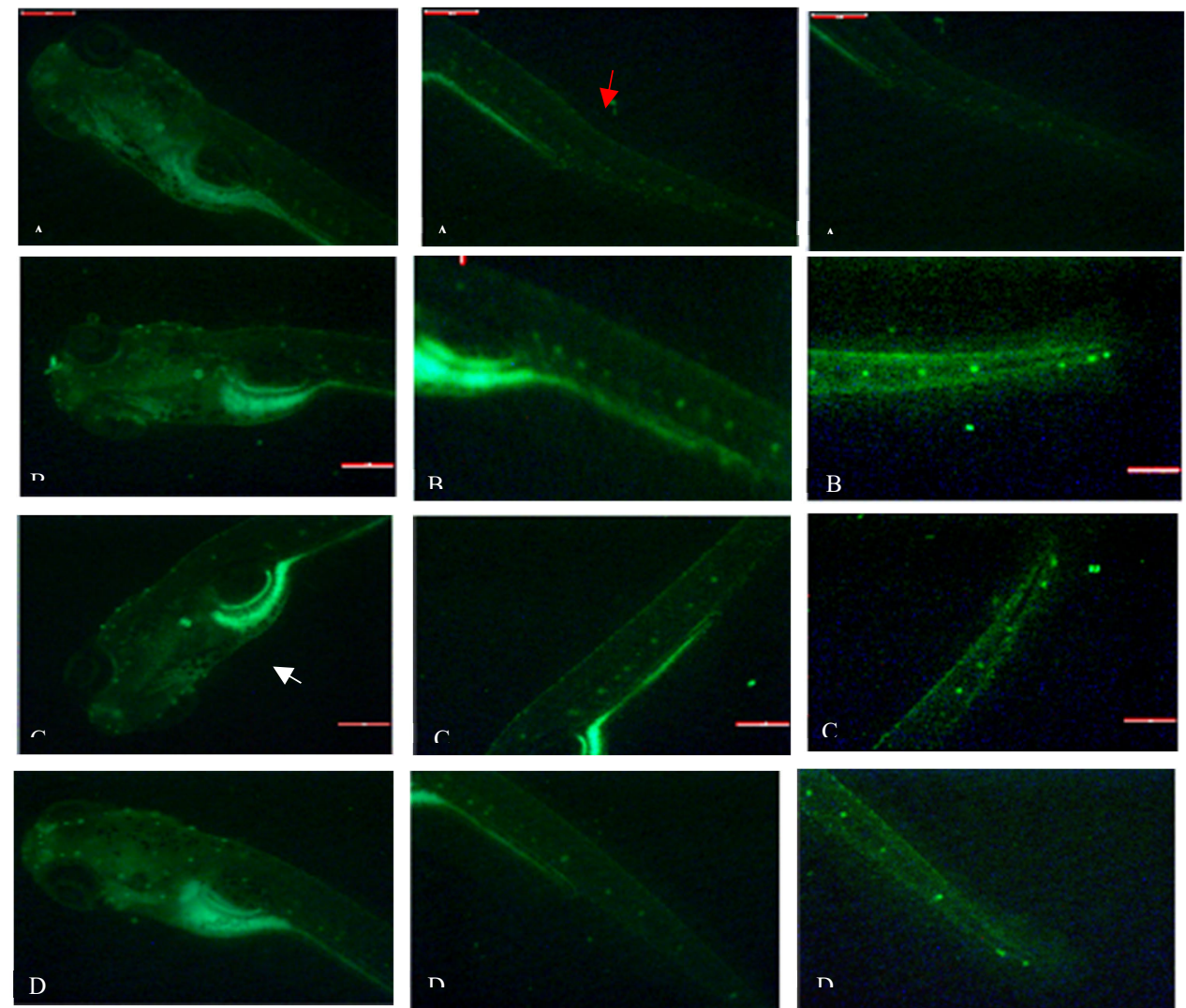

Fig. 13. Ten dpf Larva Zebrafish with 3 Hours DASPEI Staining. Under blue light exposure (A), green light exposure (B), red-light exposure (C), white light exposure (D). Head (1), body (2), and tail (3). The red arrow showed abnormality from spinal curvature, and the white arrow showed a normal digestive tract system in all treatments. Scale bar $20 \mu \mathrm{m}$.

The results of sequencing are shown in Table 4. The presence of target genes, either positive or negative, is used to describe the results in discussion. Genes 2 and 3 showed positive results in all treatments with different wavelengths (b, g, r, w). Furthermore, the gene (1) showed positive in ( $\mathrm{r}$ and $\mathrm{w}$ ), but in contrast with treatments (b and g) which showed negative results.

Table 4. DNA Sequencing in whole larval zebrafish tissue homogenates reared under with different wavelengths.

\begin{tabular}{|l|c|c|c|c|c|}
\hline \multirow{2}{*}{ Genes } & \multirow{2}{*}{ Code } & \multicolumn{4}{|c|}{ Treatments (Code) } \\
\cline { 3 - 6 } & & Blue (b) & Green (g) & Red (r) & White (w) \\
\hline opn1lw1 (LWS-1) & 1 & - & - & + & + \\
\hline mtnrlba & 2 & + & + & + & + \\
\hline aanat2 & 3 & + & + & + & + \\
\hline
\end{tabular}

(-) absent ; (+) present

opn1lw1 (lws-1) : red sensitive opsin 1

mtnrlba: melatonin receptor type 1B-A

aanat 2 : arylalkylamine $\mathrm{N}$-acetyltransferase 


\section{Discussion}

In this study, several parameters were observed for understanding the effect of different light wavelengths on growth and development in embryos and larvae in the model organism zebrafish (Danio rerio). Several parameters were observed and quantified during this study, including mortality, hatching rate, morphology effect, histological evidence of development, and expression of selected genes.

Overall, the highest survival rate occurred under green and white light (62\% followed by red $60 \%$, and blue $56 \%$ as the poorest survival rate) after 10-days of rearing. It was observed that exposure to blue and red light continued to reduce the larvae' survival rate and increase the larvae' mortality rate. Several studies had results that contrast with the present study. Villazamir et al. [49] red light exposure inhibited survival rate in 20-day post-hatching of zebrafish, in contrast with the positive effect of green light in the present study.

Villazamir et al. [49] determined that the survival rate was higher in response to blue or red light than white light exposure in European sea bass (Dientrachus labrax) within $40 \mathrm{dph}$. Below the $665 \mathrm{~nm}$ of red lighting exposure to larvae, zebrafish showed $100 \%$ mortality within 20-day post-harvest, and survival rate in $30 \mathrm{DPH}$ had a significant increase under blue light [48]. The swimbladder and pericardiac hypertrophy (edema) can induce larval mortality, causing them to float near the surface, avoid feeding, and eventually die of starvation [53].

Based on these results, only one significant difference was seen in the absorption of egg yolk under the experimental wavelength treatments. Larvae under green light had absorbed more yolk on day five compared with other treatments, possibly reflecting increased energy consumption associated with significantly advanced maturational events at that time, including eye and swimbladder differentiation. Normally the egg yolk decreases in size (diameter) during yolk absorption until the larva's mouth is fully formed and begins eating exogenously.

Using retinal photoreceptors, fish detect variations in light intensity and spectra or wavelengths [53]. In the present study, the onset of eye function was the main objective in examining the effects of different wavelengths of lighting on larval zebrafish. Photochemical exposure can cause the most severe form of retinal destruction by exposure to excessive direct sunlight and a variety of artificial light sources, such as ophthalmic instruments [16]. Fish retinas contain a duplex retina, including rods and cones that react to different wavelengths of environmental light [48]. I am not aware of studies explaining the direct effect of different light spectra on zebrafish retinal development. Based on experiment results in this study, green light showed the most favorable impact on the development of zebrafish eye diameter compared with larvae reared under blue and red light.

However, in a recent study from [31], exposure to green light-induced the development of the retina layer during the grow-out of olive flounder (Paralichthys olivaceus), containing primary photoreceptors. However, in contrast, development under red light resulted in reduced retinal photoceptor layers, suggesting physiological impairment possibly caused by stress or other physiological factors. More details are shown in Table 2.

The swimbladder is located along the body cavity between digestive organs. Swimbladder malformation is a common problem in both embryo and larval stages of fishes. Trotter et al. [42] reported that malformation of the swimbladder reached $70-100 \%$ during the larva rearing stage. Swimbladder development occurring during the larval stage in zebrafish is associated with and plays a substantial role in early survival [29]. The larval swimbladder was initially visible after hatching as a fluid-filled sac that budded from the esophagus [36], later inflated with gas. It provides buoyancy, which is important in energy-efficient larval swimming, which is of critical value in exogenous feeding.

The zebrafish swimbladder develops into a distinct 2-chamber organ and is connected to the frontal section by a pneumatic duct [36]. The measurement of the swimbladder in this 
experiment started from 4 dpf. According to Robertson et al. [36], the swimbladder differentiates from day 1 to 3 days after hatching. At the same time, the yolk is consumed and the larvae begin actively foraging, and the volume of the swimbladder increases until it has differentiated into a 2-chambered structure. Maturation and swimbladder inflation is dependent on exposure to triiodothyronine $\left(\mathrm{T}_{3}\right)$; these actions are transduced by Insulin-like Growth Factor 1 or IGF-1 [29].

Otherwise, until ten $\mathrm{dpf}$ in this experiment, the larval swimbladder remains undifferentiated, requiring as much as 24 dph fully differentiated. Swimbladders reduce body density and increase buoyancy for swimming larvae and adults [42, 36]. Increased transformation of fins, digestion, swim bladder, and the Central Nervous System also contributes to the swimbladder development in larvae, as enhanced under green light exposure and inhibited in response to blue light. The zebrafish swimbladder was observed by the presence of a black sac, indicating that it was filled with gases and presumably providing buoyancy (Fig. 3c.). Spinal deformity (kyphosis) in the zebrafish vertebrae can be a consequence of incomplete swimbladder differentiation.

As is evident in the data presented, exposure to different wavelengths of light during early development had significantly different and, in some cases, very positive and desirable impacts on initial swimbladder differentiation. Greenlight exposure had the most beneficial development-promoting effects, followed by red, blue, and white light. During early rearing, the behavior of larvae was observed visually. Under green exposure, larvae appeared to have enhanced swimming activity as compared with larvae reared under blue light. The result of this experiment is in contrast to previous studies. For example, blue light was reported to have positive impacts on fish larval development [48], although these authors pointed out that direct comparisons are not straightforward for a variety of reasons.

Relatively lower light intensity was used by [49] than in this study (750 Lux, which is severalfold more intense, as per [1]. The UK Royal SPCA explained that zebrafish hatchery light intensity is not standardized. Lower intensities are often chosen to discourage algae growth [34], so zebrafish physiology and development implications may be skewed.

The pericardium or pericardial area is the area under the fish's head where the cardiovascular system is located in teleost (Fig. 3b.). The heart is the first organ to develop, and it acts importantly in the development of vertebrate embryos. In zebrafish, early embryonic cardiac growth must perform some complex cellular and molecular mechanisms to form a mature organ capable of pumping blood [22].

Based on this study, it is established that some abnormalities occur in zebrafish larvae that are exposed to white light. White light has a negative impact on the formation of pericardial edema. Pericardial edema was described as an abnormal differentiation in the pericardium, a portion of the celomic mesodermal cavity lined and separating visceral organs, such as in the heart and wall of the body [35].

In this study, green light exposure resulted in the highest increase in body length (Fig. 12 and 13.) In agreement with our present study, Yuan et al. [54] found that body length was not impaired by green light but was impeded with red and blue light. Another result for some teleost species showed that red light significantly inhibited the growth of Gadus morhua (Atlantic cod), as evidenced by reductions in standard length and dry weight value [46]. Red light impact induces oxidative stress, causing starvation in olive flounder (Paralichthys olivaceus) [31] and reducing body length growth rate. Moreover, blue light significantly inhibited the body length and head height in European sea bass (Dicentrarchus labrax) compared to specimens responding to white, red or green exposure [53]. In contrast, some performance enhancements of rainbow trout (Oncorhynchus mykiss) cultured under blue light have been reported [19].

The retina of the newly-hatched larvae consists of an outer core layer, an inner nuclear layer, and a ganglion cell layer. Larval retinas are typically well-formed by $2 \mathrm{dph}$ and may 
not function until later [52]. The current study results indicate that green light exposure resulted in a higher percentage of full eye pigmentation in $3 \mathrm{dpf}$ zebrafish embryos. The following results are reported by [31]; green light has no negative impact on the retinal layer containing the primary photoreceptors to absorb the light. An anabolic effect of green light was reported on olive flounder by [31]. On the other hand, blue light exposure showed an inhibitory influence on eye pigmentation in embryos aged from 1-3 dpf, which agrees with Godley et al. [17], who found that blue light triggers primary retinal damage caused by mitochondrial DNA inflammation.

Fish can detect differences in light intensity with photoreceptors in the retina. In the present study, 750 lux light intensity was used. In previous studies, high light intensity has been shown to induce retinal damage in juvenile Sea Bass, Atlantic Cod, and Atlantic Salmon [53]. Red light has only mild impacts on eye pigmentation even though the percentage of fully pigmented embryos is reduced relative to those reared under green light. Nevertheless, a previous study indicates that red light has a positive impact on ovarian development by increased gonad somatic index (relative gonad weight), possibly due to the ability of red light to be absorbed and intensity decreased within increasing depth [4].

Some developmental abnormalities and malformation were observed in pericardial edema, yolk sac edema, hydrocephalus, spinal deformity, and tail deformity. Direct and indirect factors can potentially induce abnormalities and mortality; dietary variables, water temperature, salinity, pollution, rearing tank color, and lighting conditions have been implicated [53].

Under blue, red, and white lights (Fig. 12.), zebrafish exhibited higher malformation rates. Environmental variations often result in physical deformities among larval fishes. Greenlight exposure appeared to have no adverse effects on larval development in the current study. [53] determined that the highest rate of jaw malformation occurred under white or blue light and was lowest after exposure to red or green light in European Sea Bass (Dicentrarchus labrax). Another study showed that red-light exposure has a detrimental effect on Sole larvae, Solea senegalensis, resulting in developmental problems including a high proportion of jaw malformation and low survival rate [5].

The correlation between light levels and malformations has been observed in innumerable recent and older studies. Malformations can be caused by inappropriate diets such as $\beta$ carotene deficient diets, but feeding patterns change under various photoperiods and light spectra $[5,46]$. Light might play a significant role in embryogenesis; blue light wavelengths ranging from 400 to $500 \mathrm{~nm}$ (near-ultraviolet) are suspected to be potentially harmful to cells (gametes and embryos). Some previous studies have concluded that blue light is most damaging, with damage causing as much as $100 \%$ mortality in 72 hpf zebrafish embryos [44].

The lateral line systems of fish are involved in many behaviors, including predator detection and prey identification [27]. The lateral line system develops in zebrafish during the earlier embryonic and larval phase, providing sensory input necessary for feeding on plankton. The lateral line contains neuromast receptor organs on the body's surface (head, trunk, and tail) and directly interacts with the environment [21, 51]. Neuromasts are classified into two types: superficial neuromasts (skin) and neuromast canals (head and along the trunk) [27]. This study focused on the abundance of neuromasts all over the larval zebrafish body by counting to estimate the number of neuromasts.

The neuromast contains a group of hair cells with stereocilia and a kinocilium, identical to hair cell structures found in vertebrate auditory and vestibular systems that function to detect water current relative to the animal's body via movement $[27,11]$. Lateral line hair cells of zebrafish larvae are easily accessed, making them suitable for studies examining responses to experimental manipulation [20]. Zebrafish early juveniles have 20-30 neuromast patterns on the surface from the fish head to the body [30]. The adult fishes have a lateral line 
system that is more complex than the early stage, consisting of several hundred neuromasts. The lateral line is used to detect water-borne mechanical stimuli and the motion of the fish body in the water [30].

This study revealed no significant differences in response to the wavelength treatments in the resulting neuromast amount; all treatments had more than 30 neuromasts. Regarding neuromasts distributed in the head area, primary neuromasts develop from placodes on the head region, and additional neuromasts grow during development [6]. Research addressing the relationship between ambient wavelength differences and the number of neuromasts found is scarce.

The biological clock or circadian clock is the timing mechanism of an animal that induces rhythmic day/night behavioral and physiological patterns. This mechanism is manifested in the pineal, retina, brain, and mostly peripheral tissue. Photoperiod (light-dark) is an external environmental factor that induces photoreceptive tools to adapt to photoreceptor sensitivity [49]. Aanat2 and mtnrlba expression support melatonin production and photoperiod marker in vertebrates $[13,44]$. In our study, mtnrlba and aanat 2 were expressed in all wavelength treatments in response to the 14:10 LD time treatment given to all wavelength treatments. LD cycles influence a few main circadian systems, such as melatonin hormone, arylalkyamine $\mathrm{N}$-acetyltranferase (aanat), melatonin synthesis, and genes [49].

Aanat2 is a gene involved in the expression of an enzyme that controls the rate of melatonin synthesis. Melatonin production is generally inhibited under short-wavelength light exposure. Melatonin-treated zebrafish embryos grow faster, and the melatonin receptor gene mtnrlba plays a part in this. Melatonin promotes zebrafish growth by increasing cell proliferation in the embryo [44]. The arylalkylamine $\mathrm{N}$-acetyltransferase (aanat) enzyme is a melatonin contributor and a rate-limiting enzyme in melatonin production. Aanat converts serotonin to $\mathrm{N}$-acetylserotonin and catalyzes it using hydroxyindole-O-methyltransferase (HIOMT) from melatonin-by-melatonin receptors (MTs). The central nervous system and peripheral nerve networks of vertebrates are where MTs are responsible for various functions of melatonin in body tissues [53].

Positive patterns of expression of the opn 1 lwl gene were observed under red and white LED, but this gene was not expressed under blue and green LED. Color vision in vertebrates is dependent on cone photoreceptors of varying spectral intensity in the retina, and this gene is involved in the production of the red-sensitive opsin. Invertebrates, there are five main groups of photoreceptive opsins [28] :
a. RH 1 (rod opsin)
b. SWS1 (short wavelength-sensitive: blue or UV)
c. SWS2 (short wavelength-sensitive: blue),
d. RH2 (middle wavelength-sensitive: green)
e. and LWS (long-wavelength sensitive: red).

Opnllw1 functions or is expressed in developing eyes as red-sensitive cone photoreceptors in the larvae and adults [28]. Opnllwl is referred to as LWS gene; under green and blue light exposure in this study, expression of this gene was not detected. Apparently, the generation of gene products under white and red LED responded to both wavelengths (around $600 \mathrm{~nm}$, red).

\section{Conclusions and future considerations}

Greenlight produced the most favorable result for enhancing embryonic and larval zebrafish growth and development. In an apparent contradiction with some earlier reports, blue light had consistently negative impacts on nearly all parameters in this treatment, including developmental malformation and inhibition in growth (as seen in length). Expression of the 
opn1lw1 gene after exposure to light was not detected in larvae exposed to blue or green light. These gene expression results suggest that opsin required for color vision may be induced by exposure to certain wavelengths of light. Two genes involved in the synthesis of melatonin (mtnr1ba and aanat2) were active under all four experimental lighting conditions. Melatonin is secreted under conditions of darkness, and rhythmic melatonin signals are thought to act as coordinators of circadian rhythmicity. The PCR data suggest that larval melatonin rhythmicity may be established in response to photoperiod regardless of the wavelength of light. Similarly, the development of neuromasts occurred regardless of ambient light wavelengths, suggesting that lateral line function is independent of influences from the colors of environmental lighting to which our zebrafish were exposed. Other parameters were profoundly enhanced by exposure to green light (hatch rate, eye and swimbladder development, growth and survival, absence of deformities). Accelerated development under green light may have evolutionary value by enhancing larval access to favorable green water environments. Some methods and conditions used in this study may differ from previous studies, such as the light intensity used, differences in LEDs specifications between products, and possible differences in rearing different zebrafish larvae, which are not explained in detail.

\section{Acknowledgements}

Shafira Septriani led experimental activities, sample analyses, and coordination. Anley Teferra Kiddane and Gun Do Kim conducted PCR Analyses. Christopher L. Brown designed the study, supervised and participated in all laboratory and manuscript drafting.

\section{References}

1. H. Abdollahpour, B. Falahatkar, C. Lawrence, Aquac. Rep. 17 (2020)

2. B. Bagatto, B. Pelster, W.W. Burggren, J. Exp. Biol. 204, 9 (2001)

3. M.K. Bairwa, N. Saharan, K.D. Rawat, V.K. Tiwari, K.P. Prasad, Fish. Technol. 54, 6 (2017)

4. M.A.J. Bapary, M.N. Amin, Y. Takeuchi, A. Takemura, Aquac. 314, 5 (2011)

5. B. Blanco-Vives, N. Villamizar, J. Ramos, M.J. Bayarri, O. Chereguini, F.J. SánchezVázquez, Aquac. 306, 9 (2010)

6. J.H. Blaxter, L.A. Fuiman, Function of the free neuromasts of marine teleost larvae (Springer-Verlag, New York, 1989)

7. G. Boeuf, P.Y. Le Bail, Aquac 177, 23 (1999)

8. A. Brooks, M. Waters, Nat. Rev. Endocrinol. 6, 10 (2010)

9. L. F. Canosa, J.P. Chang, R.E. Peter, Gen. Comp. Endocrinol. 151, 26 (2007)

10. W. Choi, A. Hamidoghli, J. Bae, S. Won, Y.H. Choi, K.W. Kim, S.C. Bai, J. Fish Aquat. Sci. 23, 8 (2020)

11. S. Coombs, C.B. Braun, B. Donovan, J. Exp. Biol. 204, 11 (2001)

12. W. Dawson, T. Nakanishi-Ueda, D. Armstrong, D. Reitze, D. Samuelson, M. Hope, R. Koide, Exp. Eye Res. 73, 10 (2001)

13. N.H. De Borsetti, B.J. Dean, E.J. Bain, J.A. Clanton, R.W. Taylor, J.T. Gamse, Dev. Biol. 358, 10 (2011)

14. T. Ellis, B. North, A. P. Scott, N.R. Bromage, M. Porter, D. Gadd, J. Fish Biol. 61, 38 (2002) 
15. B. Fraysse, R. Mons, Garric, J. Ecotox. Environ. Safe 63, 13 (2006)

16. M.I.G. Mohesh, M. I., Latha, R., Chitra, M. Natl. J. Physiol. 5, 3 (2017)

17. B.F. Godley, F.A. Shamsi, F.Q. Liang, S.G. Jarrett, S. Davies, M. Boulton, J. Biol. Chem. 280, 6 (2005)

18. C. Graham, M.A. von Keyserlingk, B. Franks, Appl. Anim. Behave. Sci. 200, 9 (2018)

19. U. Güller, S. Önalan, M. Arabac, B. Karataş, M. Yaşar, O.I. Küfrevioğlu, Fish Physiol. Biochem, 12 (2020)

20. J.A. Harris, A.G. Cheng, L.L. Cunningham, G. MacDonald, D.W. Raible, E.W. Rubel, JARO. 4, 15 (2003)

21. P.P. Hernández, V. Moreno, F.A. Olivari, M.L. Allende, Hear. Res. 213, 10 (2006)

22. Y. Huang, Z. Chen, Y. Meng, Y. Wei, Z. Xu, J. Ma, H. Lu, Ecotox. Environ. Safe 205, $9(2020)$

23. B.G. Kim, C.L. Brown, Am. Zool. 37, 11 (1997)

24. C. Kistler, D. Hegglin, H. Würbel, B. König, Appl. Anim. Behave. Sci. 135, 9 (2011)

25. C. Lawrence, Aquac. 269, 20 (2007)

26. V. Lugert, G. Thaller, J. Tetens, C. Schulz, Krieter, J. Rev. Aquacult. 8, 12 (2016)

27. P.J. Mekdara, M.A. Schwalbe, L.L. Coughlin, E.D. Tytell, J. Exp. Biol. 221, 1-12 (2018)

28. D.M. Mitchell, C.B. Stevens, R.A. Frey, S.S. Hunter, R. Ashino, S. Kawamura, D.L. Stenkamp, PLoS Genet. 11, 8 (2015)

29. M.H.R. Molla, M.T. Hasan, W.J. Jang, C.D.S. Diaz, P. Appenteng, H. Marufchoni, B. Jahan, C.L. Brown, Aquac. Res. 50, 9 (2019)

30. S.L. Murakami, L.L. Cunningham, L.A. Werner, E. Bauer, R. Pujol, D.W. Raible, E.W. Rubel, Hear. Res. Developmental 186, 9 (2003)

31. R.B. Ndanda, Y.R. Kim, J.M. Kim, J. Life Scie. 29, 7 (2019)

32. C. Nusslein-Volhard, R. Dahm, Zebrafish (Oxford University Press, Oxford, 2002)

33. D.T. Organisciak, D.K. Vaughan, Prog. Retin. Eye Res. 29, 21 (2010)

34. B. Reed, M. Jennings, Guidance on the housing and care of zebrafish, Danio rerio (RSPCA, West Sussex, 2010)

35. M.J. Reimers, J.K. La Du, C.B. Periera, J. Giovanini, R.L. Tanguay, Neurotoxicol. Teratol. 28, 11 (2006)

36. G.N. Robertson, C.A.S. McGee, T.C. Dumbarton, R.P. Croll, F.M. Smith, J. Morphol. 268, 18 (2007)

37. R.F. Rohlf, Morphometrics 21, 17 (1990)

38. R. Shinoda, H. Wu, M. Murata, H. Ohnuki, Y. Yoshiura, H. Endo, Sensors and Actuators: B Chem. 247, 8 (2017)

39. R. Spence, G. Gerlach, C. Lawrence, C. Smith, Biol. Rev. 83, 21 (2008)

40. S.A. Strungaru, G. Plavan, A. Ciobica, M. Nicoara, M.A. Robea, C. Solcan, Petrovici, Ecotox. Environ. Safe 171, 854-862 (2019)

41. P.S. Suriyampola, D.S. Shelton, R. Shukla, T. Roy, A. Bhat, E.P. Martins, Zebrafish 13, 8 (2016)

42. A.J. Trotter, P.M. Pankhurst, P.R. Hart, Aquac. 198, 13 (2001)

43. B. Tsang, H. Zahid, R. Ansari, R.C.Y. Lee, A. Partap, R. Gerlai, Zebrafish 14, 12 (2017)

44. U.V. Üstündağ, E. Çalıskan-Ak, P.S. Ateş, I. Ünal, G. Eğilmezer, T. Yiğitbaşı, EmekliAlturfan, E. Sci. Rep. 9, 12 (2019) 
45. L.M. Vera, H. Migaud, Aquac. 296, 9 (2009)

46. N. Villamizar, A. García-Alcazar, F.J. Sánchez-Vázquez, Aquac. 292, 7 (2009)

47. N. Villamizar, B. Blanco-Vives, H. Migaud, A. Davie, S. Carboni, F.J. SanchezVazquez, Aquac. 315, 9 (2011)

48. N. Villamizar, L.M. Vera, N.S. Foulkes, F.J. Sánchez-Vázquez, Zebrafish 11, 9 (2014)

49. J. Wan, D. Goldman, Curr. Opin. Genet. Dev. 40, 7 (2016)

50. J.F. Webb, J.E. Shirey, Dev. Dyn. 228, 15 (2003)

51. L. Wu, M. Han, Z. Song, S. Xu, J. Li, X. Li, Fish Shellfish Immunol. 90, 9 (2019)

52. H. Yan, Q. Liu, X. Cui, X. Shen, P. Hu, W. Liu, Y. Liu, Aquac. Res. 50, 14 (2019)

53. S.S. Yuan, H.Z. Xu, L.Q. Liu, J.L. Zheng, Fish Physiol. Biochem. 43, 2 (2017)

\section{Appendix}

\subsection{DNA extraction}

1. Make ready an ice with bucket

2. Make ready genomic DNA extraction kit with

- Collection tube

- Spin column

- Buffers - TL (tissue lysis), GB (gene binding), GW1 ethanol added, GW2 ethanol added

3. Prepare absolute ethanol $(200 \mu \mathrm{L}$ x number of sample $)+30 \mu \mathrm{L}$

4. Prepare Proteinase-K $(20 \mu \mathrm{L}$ for each sample)

5. Turn on the heat-block machine at $56^{\circ} \mathrm{C}$

6. Prepare Three $1.5 \mathrm{~mL}$ Eppendorf tube for each sample and label it

7. Prepare Ultra distilled water $100 \mu \mathrm{L}$ x number of sample

8. Prepare scissor and twizzer

9. Prepare $50 \mathrm{mLconical}$ tube for discharge waste water

10. Sample preparation - keep the sample in the ice in $-80^{\circ} \mathrm{C}$

11. Clean twizzer with alcohol and tissue

12. Weigh empty $1.5 \mathrm{~mL}$-eppenderoff

13. Take and weight zebrafish sample tissue (20-100 mg) and put it into $1.5 \mathrm{ml}$ Eppendorf and give label to each Eppendorf

14. Clean the twizzer with alcohol and tissue paper for every sample, for contamination prevention.

15. Add $200 \mu \mathrm{L}$ TL-buffer in each sample and vortex a little.

16. Vortex proteinase- $\mathrm{K}$ and add $20 \mu \mathrm{L}$ proteinase- $\mathrm{K}$ in each sample and vortex a little.

17. Put in the heat block machine with $56^{\circ} \mathrm{C}$ for $20-60$ minutes, then check for every minute and vortex a little, and put back to a heat-blocker until fully dissolved.

18. Centrifuge at 13,200 RPM for 3 minutes.

19. Transfer $200 \mu \mathrm{L}$ supernatant in a new $1.5 \mathrm{~mL}$ Eppendorf, and then discard the pellet.

20. Add $200 \mu \mathrm{L}$ GB-buffer into the collected supernatant (careful for contamination) and then vortex.

21. Incubate in a $56^{\circ} \mathrm{C}$ heat-blocker machine for 10 minutes.

22. Turn-off the heat blocker machine.

22. Add $200 \mu \mathrm{L}$ absolute ethanol in each incubated samples (careful for tip contamination) and vortex a little. 
23. Transfer $600 \mu \mathrm{L}$ to spin-column by pipette. (Careful for contamination and do not touch the spin-column membrane by pipette tip)

24. Centrifuge at 13,200 RPM for 1 minute.

25. Prepare new collection tube.

26. Transfer spin-column to new collection tube and discard the fluid.

27. Add 500 $\mu \mathrm{L}$ GW1 and centrifuge at 13,200 RPM for 1 minute.

28. Prepare new collection tube.

31. Transfer spin-column to new collection tube and discard the fluid.

32. Add $500 \mu \mathrm{L}$ GW2 and centrifuge at 13200 RPM for 1 minute.

33. Prepare new collection tube.

34. Transfer spin-column to new collection tube and discard the fluid.

35. Centrifuge at 13200 RPM for 1 minute.

36. Prepare new $1.5 \mathrm{~mL}$-eppenderoff tube and label.

37. Transfer the spin-column to a $1.5 \mathrm{~mL}$-eppenderoff and label.

38. Drop $100 \mu \mathrm{L}$ ultra-distilled water at the middle of the membrane. (do not touch the membrane of spin column)

39. Stays 1-2 minutes at room temperature.

40. Centrifuge at 13200 RPM for 1 minute.

41. Discard the spin-column, collect DNA and keep it in the refrigerator.

42. Clean and replace the materials.

\subsection{PCR (Polymerase Chain Reaction)}

1. Prepare the primer for each gene forward and reverse

2. Add $150 \mu \mathrm{L}$ ultra-distilled water to each primer

3. Prepare working solution with ratio dilution $1: 10: 10 \mu \mathrm{L}$ primer add $90 \mu \mathrm{L}$ ultradistilled water and put into $1.5 \mathrm{~mL}$ Eppendorf

4. Vortex 10 seconds

5. Give label and code:

1: opn 1 lw1.

2: mtnrlba

3: aanat2

b: blue

g: green

r: red

w: white

6. Prepare 12 premix, spin and label it

7. Add $7 \mu \mathrm{L}$ ultra-distilled water in each premix

8. Add $1 \mu \mathrm{L}$ primer in each different premix

9. Add $1 \mu \mathrm{L}$ DNA in each premix

10. Spin the premix to mix

11. Turn on the thermal cycler machine (PCR machine) and choose the method

12. Put the mixed premix in the thermal cycler machine and start the PCR process. It takes around 2 hours

\subsection{Electrophoresis}

1. Start to making gel for electrophoresis

2. Weigh 0.3gram agarose

3. Put the agarose in the Erlenmeyer glass

4. Add $20 \mathrm{~mL}$ TAE (Tris-acetate-EDTA) x number of sample and shake 
5. Put in the microwave for 1 minute and shake

6. Add $1 \mu \mathrm{L}$ Ecodye per $20 \mathrm{~mL}$ TAE

7. Shake it and pour dilute agarose in the cassette

8. Make sure no bubble formation in the agar or push and discard the bubble by using glass stick

9. Put the comb in the agarose fluid and wait for 20-30 minutes till it turns to gel

10. After 20-30 minutes remove the comb from the gel

11. Clean the bottom and side of the cassette by alcohol and tissue paper

12. Put gel in the electrophoresis machine and put more TAE until the gel fully submerged

13. Prepare $100 \mathrm{bp}$ (base pair) DNA-ladder kit

14. Add $2 \mu \mathrm{L} 100$ bp DNA-ladder kit in the first gel well

15. Add $2 \mu \mathrm{L}$ extracted DNA in each gel well, keep premix DNA for PCR purification

16. Close the electrophoresis system

17. Turn on the electrophoresis system and run for 30 minutes

18. After 30 minutes, first turn off the electrophoresis system

19. Transfer the gel to the illuminator machine (vilber lourmat)

20. Then check the bands formed on the gel and take a picture

\subsection{PCR Purification}

1. Make ready DNA with premix

2. Make ready PCR purification kit with : PCR-B buffer, pw buffer with ethanol added, spin column and collection tubes

3. Prepare ultra-distilled water

4. Make ready $50 \mathrm{~mL}$ conical tube and tissue paper for waste collection

5. Make read $1.5 \mathrm{~mL}$ Eppendorf tube and give detail code

6. Spin the DNA with premix

7. Add $100 \mu \mathrm{L}$ PCR-B buffer into DNA premix, mixing with pippet the transfer all drop into in the middle of spin column membrane '

8. Centrifuge at $13200 \mathrm{rpm}$ for 1 minute

9. After centrifugation, discard the fluid from collection tube

10. Add $500 \mu \mathrm{L}$ pw buffer in spin column

11. Centrifuge at $13200 \mathrm{rpm}$ in 1 minute

12. Discharge the fluid from collection tube

13. Again add $500 \mu \mathrm{L}$ pw buffer in spin column

14. Centrifuge at $13200 \mathrm{rpm}$ in 1 minute

15. Transfer the spin column to labeled $1.5 \mathrm{~mL}$ Eppendorf tube

16. Add $20 \mu \mathrm{L}$ distilled water at the middle spin-column membrane

17. Stay for 1-2 minutes

18. Centrifuge at $13200 \mathrm{rpm}$ for 1 minute

19. Discard spin-column and keep purified DNA with Eppendorf in $-20^{\circ} \mathrm{C}$ refrigerator and do sequencing in company service. 